\title{
Hybrid technique of cortical bone trajectory and pedicle screwing for minimally invasive spine reconstruction surgery : A technical note
}

\author{
Yoichiro Takata, Tetsuya Matsuura, Kosaku Higashino, Toshinori Sakai, \\ Takuya Mishiro, Naoto Suzue, Hirofumi Kosaka, Daisuke Hamada, Tomohiro Goto, \\ Toshihiko Nishisho, Yuichiro Goda, Ryosuke Sato, Takahiko Tsutsui, Ichiro Tonogai, \\ Fumitake Tezuka, Kazuaki Mineta, Tetsuya Kimura, Akihiro Nitta, Tadahiro Higuchi, \\ Shingo Hama, and Koichi Sairyo \\ Department of Orthopedics, the University of Tokushima, Tokushima, Japan
}

\begin{abstract}
The pedicle screw (PS) system is widely used for spinal reconstruction. Recently, screw insertion using the cortical bone trajectory (CBT) technique has been reported to provide increased holding strength of the vertebra, even in an osteoporotic spine. CBT is also beneficial due to its low invasiveness. We have been performing hybrid reconstruction with CBT at the cranial level and PS at the caudal level based on the concept of minimal invasiveness. We applied this hybrid technique to 6 cases of degenerative spondylolisthesis. Surgery was completed with a small skin incision of around 5-6 cm, which is shorter than that of the conventional PS procedure. The mean percent slippage before surgery was $19.8 \%$, and this was reduced to $3.9 \%$ after surgery and almost maintained 3 months after surgery. Furthermore, no major surgical complications were observed. Here, we introduce the minimally invasive hybrid technique of CBT-PS. Surgeons should be aware of the procedure as an option for minimally invasive lumbar spine reconstructive surgery. J. Med. Invest. 61 : 388-392, August, 2014
\end{abstract}

Keywords : lumbar degenerative spondylolisthesis, pedicle screw, cortical bone trajectory, fusion surgery

\section{INTRODUCTION}

Santoni et al. (1) proposed in 2009 a new lumbar spine reconstruction technique called the cortical bone trajectory (CBT) technique. This technique differs from the conventional pedicle screw (PS) technique in which a screw is inserted through the anatomical axis of the pedicle into the vertebral

Received for publication May 7, 2014 ; accepted June 4, 2014.

Address correspondence and reprint requests to Yoichiro Takata, MD, PhD, Department of Orthopedics, the University of Tokushima, 3-18-15 Kuramoto, Tokushima 770-8503, Japan and Fax : +81-88-633-0178. body. In the CBT technique, a screw follows a sagittal caudocephalad path and a laterally directed path in the transverse plane to maximally engage the cortical bone from the pedicle to the vertebral body. Santoni et al. (1) measured the pullout strengths of the conventional PS and new CBT technique and found a $30 \%$ increase in uniaxial yield pullout load when employing CBT. Thus, CBT screwing is especially beneficial for osteoporotic spine in elderly patients.

The CBT technique also has other advantages due to the screw insertion point. The screw insertion through a medial starting point may minimize damage to the paravertebral muscles, facet joint, 
and capsule. Especially on the cranial side of fusion, a conventional PS needs to be inserted laterally from the facet joint so that the screw insertion maneuver will not damage the facet joint and capsule. Thus, to insert a conventional PS at the cranial level of fusion, the paravertebral muscles need to be dissected laterally to the transverse process including the entire lamina. However, the CBT technique at the cranial level requires soft tissue dissection at only the inferior part of the lamina. Similarly, at the caudal level of fusion, CBT needs soft tissue dissection at the inferior lamina. However, PS at the caudal level can be inserted without any further dissection after decompression and facetectomy.

Because of the low invasiveness of CBT at the cranial level, we have started to perform a hybrid technique with CBT at the cranial level and PS at the caudal level (Figure 1). To date, only a few studies have reported on the CBT procedure, and to the best of our knowledge, very few studies have reported on the hybrid CBT-PS technique. In this paper, we introduce our hybrid technique as a technical note and review the clinical and radiological outcomes of the initial 6 cases of degenerative spondylolisthesis.

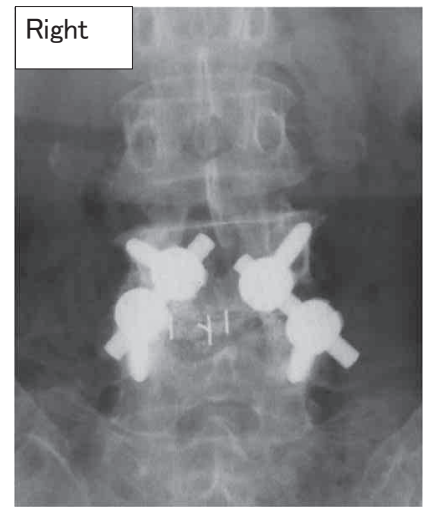

Antero-posterior view

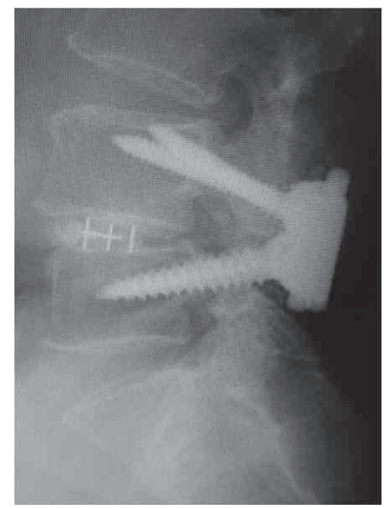

Lateral view
Figure 1 : Representative radiographs after the hybrid cortical bone trajectory-pedicle screw (CBT-PS) technique. Two screws inserted by CBT at L4 and 2 conventional PS inserted at L 5 are shown. Note the difference in the insertion direction in each procedure.

\section{SURGICAL TECHNIQUE}

We explain the surgical technique in a representative case of $\mathrm{L} 4$ spondylolisthesis. A skin incision of about $5-6 \mathrm{~cm}$ is made around the interlaminar area at L4-5 (Figure 2). Decompression including L4 laminotomy, L4-5 flavectomy, L5 laminotomy,

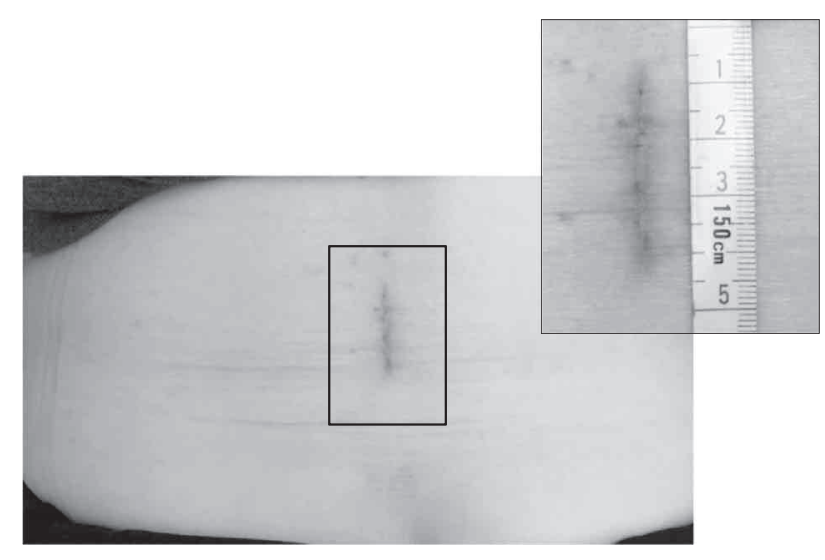

Figure 2 : Incisional skin scar in a patient who underwent CBTPS.

and single-sided facetectomy is performed. A cage is inserted by the transforaminal interbody fusion technique. At L4, 2 screws are inserted by the CBT technique on each side, and at L5, 2 PS are inserted according to the conventional technique proposed by Roy-Camille et al. (2). Unlike Weinstein's technique for PS insertion (2), Roy-Camille's technique does not require paravertebral dissection at the transverse process. Figure 3 shows the insertion point with both PS insertion techniques. The dotted lines indicate the pathway for Roy-Camille's technique and the straight lines indicate that for Weinstein's technique.

The insertion point of the CBT screw is determined on an anteroposterior radiological view using a $\mathrm{C}$-arm image intensifier. For the right and left

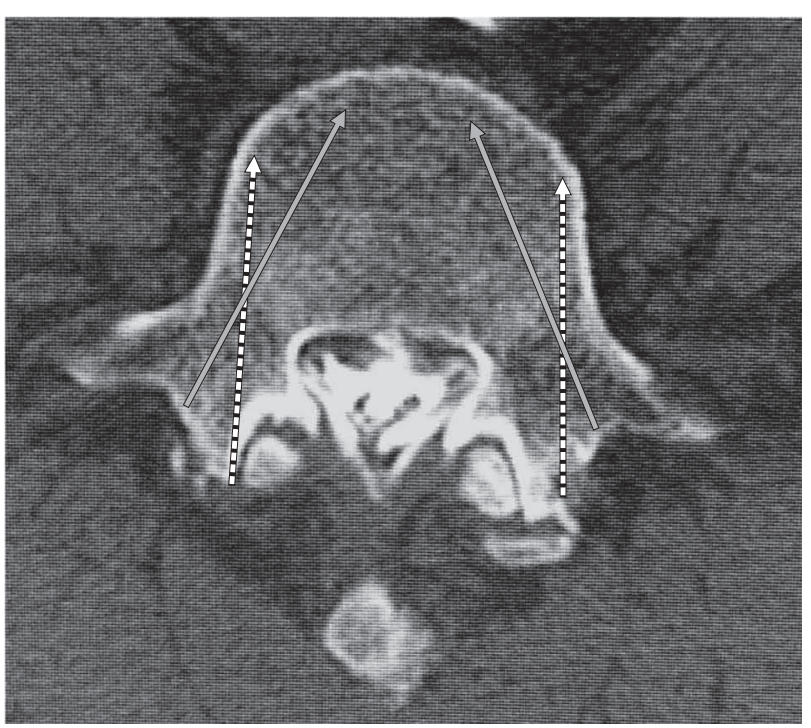

Figure 3 : Insertion point and pathway in each kind of pedicle screwing technique. Dotted lines indicate the pathway for RoyCamille's technique and straight lines indicate that for Weinstein's technique. 
CBT screw insertion, the points are at 7 o'clock and 5 o'clock of the pedicle, respectively (Figure 4). This is similar to the technique proposed by Matsukawa et al. (3). The lateral view on the image intensifier is used to understand the sagittal trajectory of CBT screwing (Figure 5). First, the cortex at the insertion point is drilled. Then, a pedicle probe is inserted while observing the lateral view on the image intensifier. For the laterally directed path in the transverse plane, screw insertion is performed at $10 \mathrm{de}-$ grees to the insertion angle in the medial to lateral direction.

To reduce slippage in this series, we used the MESA $^{\circledR}$ system (K2M Inc., Leesburg, VA). The postsurgical radiographs in Figure 1 show that the CBT screws at LA follow a caudocephalad path in the sagittal plane and a laterally directed path in the transverse plane. On the other hand, the screws at L5 are inserted along the anatomic axis of the pedicle into the vertebral body.

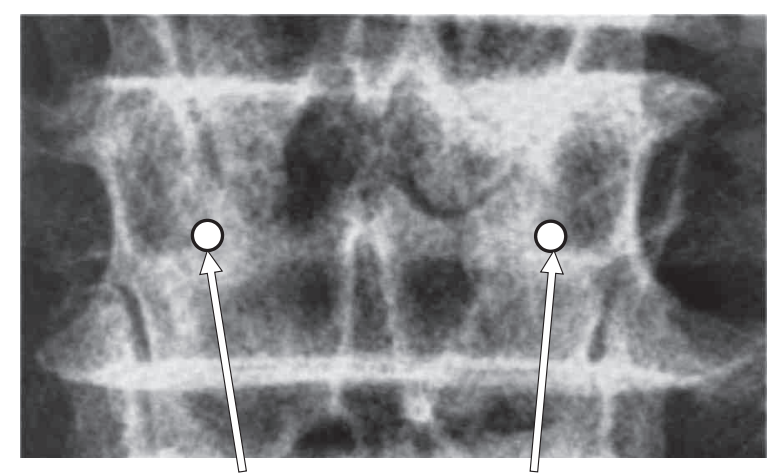

Rt insertion point

Rt insertion point

Figure 4 : Insertion point of the CBT screw in the anteroposterior radiological view on the $\mathrm{C}$-arm image intensifier. For the right and left $\mathrm{CBT}$ screw insertion, the points are at $7 \mathrm{o}$ 'clock and 5 o'clock of the pedicle, respectively.

\section{METHOD}

Six cases of degenerative spondylolisthesis were treated with this hybrid CBT-PS technique. The affected vertebra was L3 in 1 case and LA in the remaining 5 cases. All patients were women, aged $55,65,72,75,76$, and 77 years. Clinical outcome was evaluated by Japanese Orthopaedic Association (JOA) score (4). Radiological outcome was evaluated by comparing the percent slippage before surgery with that immediately after surgery and 3 months later.

\section{RESULTS}

The mean operation time was $175.8 \mathrm{~min}$ (150-200 min). Blood loss was $70-200 \mathrm{mg}$, and no patients required blood transfusion. In addition, there were no surgical complications such as dural tear, cauda equina injury, nerve root injury, or deep surgical site infection requiring removal of the spinal implants. Only 1 patient had a mild infection of unknown origin 6 weeks after the surgery, in which the white blood cell count and C-reactive protein (CRP) level increased to 9800 cells $/ \mu \mathrm{L}$ and $0.7 \mathrm{mg} /$ $\mathrm{dL}$, respectively. The infection required antibiotics for 2 months ; however, it resolved without needing to remove the instrumentation. The mean JOA score was 15.5 before surgery and improved to 24.5 after the surgery.

Figure 6 shows the time course of the percent slippage in each case. The slippage was reduced from $19.8 \%$ to $3.9 \%$ using The MESA ${ }^{\circledR}$ system, and the reduced position was maintained 3 months later. Figures 7 and 8 show 2 cases treated by this procedure. In both cases, slippage was reduced to

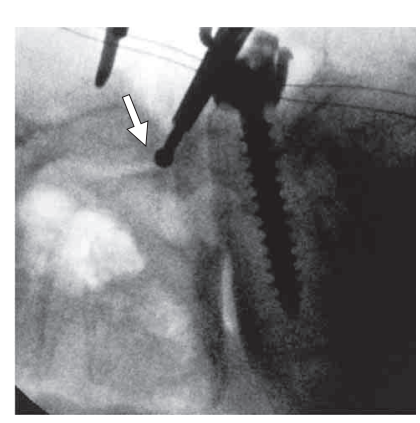

(A)

Drilling of the dorsal surface of the lamina

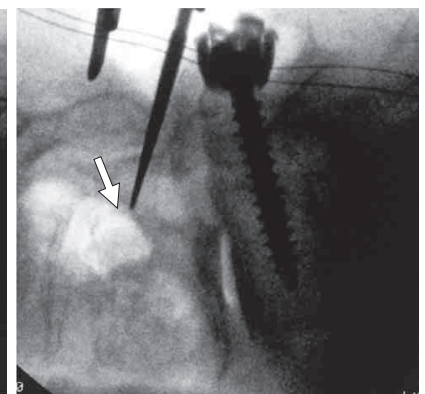

(B)

Proving inside the pedicle wall

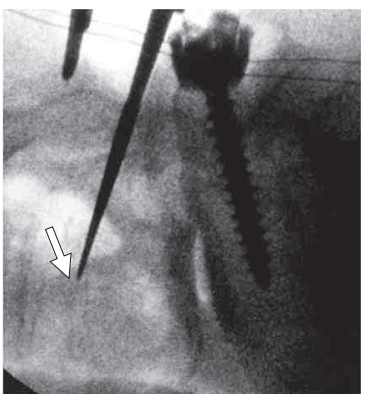

(c)

Proving inside the vertebral body

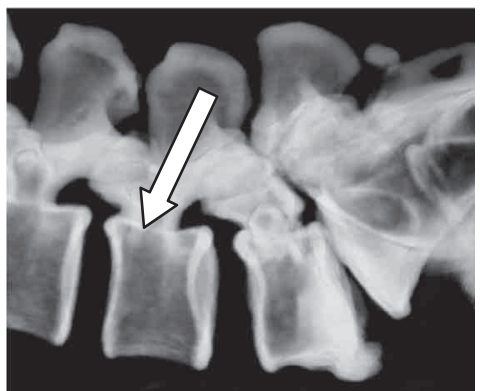

(D)

Schematic illustration of insertion of CBT screw

Figure 5 : Lateral view on the image intensifier during preparation for CBT. After drilling the insertion point in the cortex, a pedicle probe is inserted while looking at the lateral view on the image intensifier. 


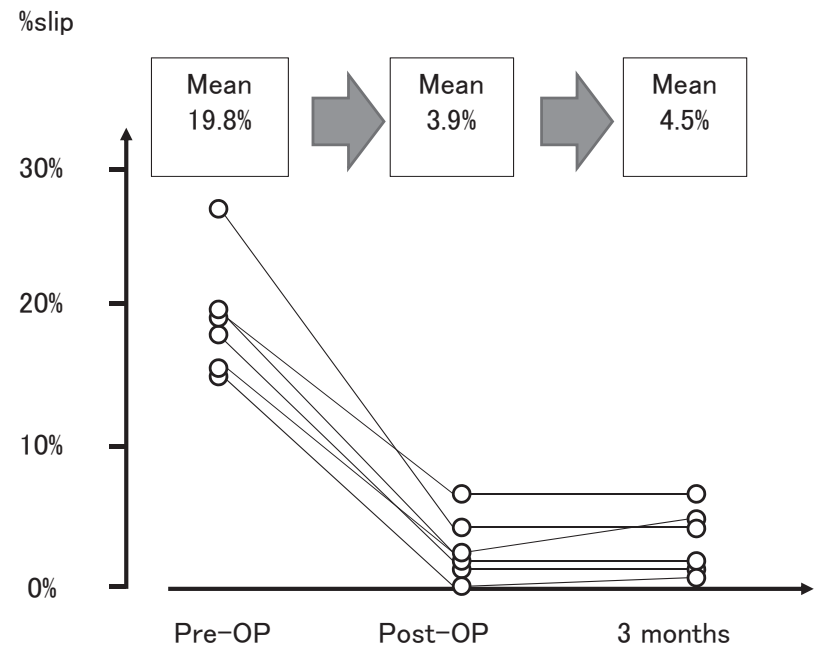

Figure 6 : Time course of percent slippage in each case. The mean percent slippage was reduced with the MESA ${ }^{\circledR}$ system from $19.8 \%$ to $3.9 \%$ after the surgery and maintained for 3 months after the surgery.

\section{CBT \#5 78 y.o. L4 spondylolisthesis}

Pre-OP

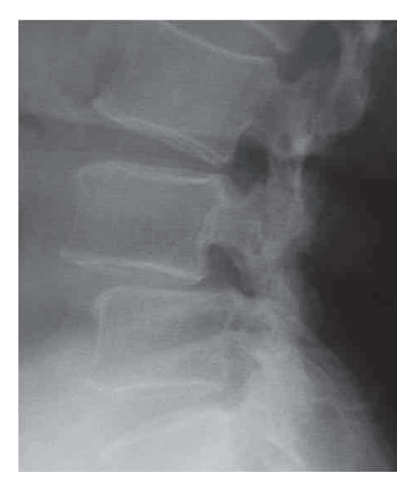

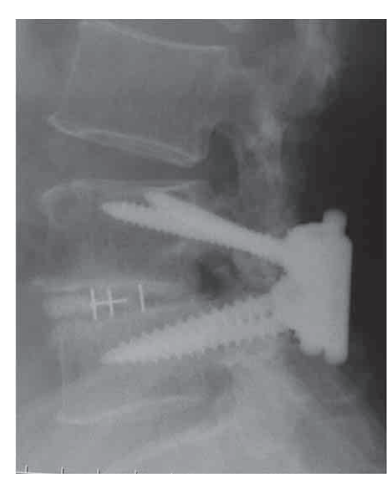

$3 \mathrm{M}$ after
Figure 7 : Plain radiographs of a 78 -year-old patient before and 3 months after the surgery. Note the reduced position achieved with the CBT-PS technique.

CBT \#5 72y.o. L4 spondylolisthesis

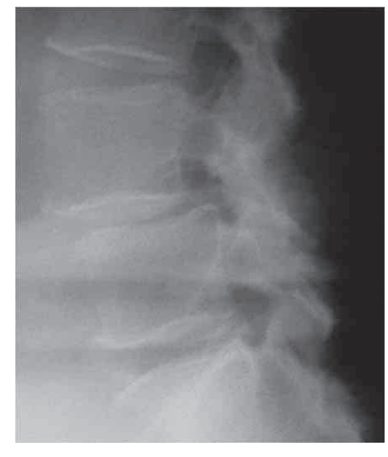

Pre-OP

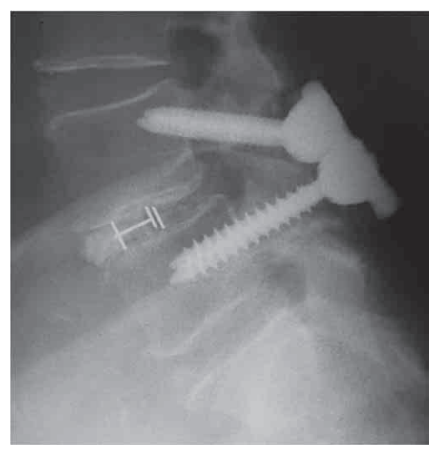

$3 \mathrm{M}$ after
Figure 8 : Plain radiographs of a 72-year-old patient before and 3 months after the surgery. Note the reduced position achieved with the CBT-PS technique. almost the normal position, which was maintained for 3 months.

\section{DISCUSSION}

\section{Minimal invasiveness :}

In theory, the present procedure is less invasive than conventional PS fusion surgery. At our institution, conventional single-level transforaminal lumbar interbody fusion surgery with PS requires a skin incision of around $8-10 \mathrm{~cm}$, whereas this hybrid CBT-PS procedure requires an incision of $5-6 \mathrm{~cm}$. In terms of incision length, our technique is less invasive than the conventional PS technique.

To understand the invasiveness to bone and muscle during the back surgery, CRP, creatinine kinase (CK), and interleukin-6 values have sometimes been evaluated (5-7). However, in this technical note, we need to concentrate on the technical note. Presently, we have initiated to understand its minimally invasiveness with evaluating these parameters.

Comparison with the other minimally invasive fusion techniques :

Use of the percutaneous pedicle screwing (PPS) procedure is another option for minimally invasive fusion surgery. Although the midline skin incision in PPS can be smaller, 2 additional skin incisions (and as many as 4) are needed. Furthermore, in the PPS procedure, the PS must pass through the paravertebral muscles, which may damage them, although the CK level after surgery is reported to be lower than that with the conventional PS technique (8). As a next step in examining invasiveness, we need to compare the invasiveness of the CBT technique with that of the PPS technique.

\section{Repositioning and holding power with our hybrid CBT-PS technique :}

We applied the hybrid CBT-PS technique to patients with degenerative spondylolisthesis. The mean percent slippage was $19.8 \%$ before surgery and 3.9\% after surgery, and was maintained at $4.5 \%$ at the 3 month follow up, thus indicating sufficient holding strength of the slipped vertebra by CBT screwing. Compared with reports of the PS system, our reduction ratio using the hybrid CBT-PS technique would be similar to that of the conventional PS procedure. All patients were over 50 years old, and 3 were over 70 years old and likely had an osteoporotic spine. Matsukawa et al. (3) recently measured the final 
insertional torque (IT), which is reported to be a good indicator of screw pullout strength, in the PS and CBT techniques and found that the IT of CBT was about 2 -fold that of PS. Thus, it is reasonable to regard the holding strength of the vertebral body with CBT screwing in our cases to be in good agreement with the previous biomechanical data reported by Santoni et al. (1) and Matsukawa et al. (3).

\section{Future investigation :}

Santoni et al. first described the CBT technique in 2009 (1) but reported only biomechanical not clinical data. Moreover, only a few studies have reported the biomechanical, clinical, and radiological outcomes in actual surgical cases $(3,9)$. Therefore, the data on this technique are as yet limited. In the future, comprehensive clinical and radiologic data are needed to clarify the efficacy of this theoretically ideal and minimally invasive screwing technique, which we believe has the potential to become the gold standard for minimally invasive spinal fusion surgery.

\section{REFERENCES}

1. Santoni BG, Hynes RA, McGilvray KC, Rodriguez-Canessa G, Lyons AS, Henson MA, Womack WJ, Puttlitz CM : Cortical bone trajectory for lumbar pedicle screws. Spine J 9(5) : 366-73, 2009

2. Weinstein JN, Rydevik BL, Rauschning W : Anatomic and technical considerations of pedicle screw fixation. Clin Orthop Relat Res (284) : 34-46, 1992
3. Matsukawa K, Yato Y, Kato T, Imabayashi H, Asazuma T, Nemoto $\mathrm{K}$ : In vivo analysis of insertional torque during pedicle screwing using cortical bone trajectory technique. Spine (Phila $\mathrm{Pa}$ 1976) 39(4) : E240-5, 2014

4. Japanese Orthopaedic Association : Japanese Orthopaedic Association Assessment Criteria Guidelines Manual. Tokyo : JOA ; 1996 : 46-49.

5. Pan L, Zhang P, Yin Q. Comparison of tissue damages caused by endoscopic lumbar discectomy and traditional lumbar discectomy : A randomised controlled trial. Int J Surg. 2014 Feb 28. pii : S1743-9191(14)00054-5.

6. Sasaoka R1, Nakamura H, Konishi S, Nagayama R, Suzuki E, Terai H, Takaoka K : Objective assessment of reduced invasiveness in MED. Compared with conventional one-level laminotomy. Eur Spine J 15(5) : 577-82, 2006

7. Huang TJ, Hsu RW, Li YY, Cheng CC : Less systemic cytokine response in patients following microendoscopic versus open lumbar discectomy. J Orthop Res 23(2) : 406-11, 2005

8. Lehmann W, Ushmaev A, Ruecker A, Nuechtern J, Grossterlinden L, Begemann PG, Baeumer T, Rueger JM, Briem D : Comparison of open versus percutaneous pedicle screw insertion in a sheep model. Eur Spine J 17(6) : 857-63, 2008

9. Matsukawa K, Yato Y, Nemoto O, Imabayashi H, Asazuma T, Nemoto K: Morphometric Measurement of Cortical Bone Trajectory for Lumbar Pedicle Screw Insertion Using Computed Tomography. J Spinal Disord Tech 26 : E248-E253, 2013 